\section{Comportamentos em saúde e adoção de medidas de proteção individual durante a pandemia do novo coronavírus: iniciativa ELSI-COVID-19}

\section{Health behaviours and the adoption of individual protection measures during the new coronavirus pandemic: the ELSI-COVID-19 initiative}

\section{Comportamientos en salud y adopción de medidas de protección individual durante la pandemia del nuevo coronavirus: la iniciativa ELSI-COVID-19}

Sergio Viana Peixoto 1,2

Mary Anne Nascimento-Souza 1

Juliana Vaz de Melo Mambrini 1

Fabiola Bof de Andrade 1

Deborah Carvalho Malta 2

Maria Fernanda Lima-Costa 1,3

doi: 10.1590/0102-311X00195420

\title{
Resumo
}

O objetivo deste estudo foi avaliar se os comportamentos saudáveis determinam a adoção de medidas protetivas individuais para o combate à COVID-19. Os dados foram obtidos da iniciativa ELSI-COVID-19, inquérito telefônico conduzido entre os participantes do Estudo Longitudinal da Saúde dos Idosos Brasileiros (ELSI-Brasil), que inclui amostra nacional representativa da população com 50 anos ou mais. Os desfechos avaliados foram três medidas protetivas (não ter saído de casa na última semana, usar máscara quando saiu de casa e higienizar as mãos quando retornou para casa) e as variáveis explicativas foram os comportamentos em saúde (tabagismo, consumo de bebidas alcoólicas, consumo de frutas e hortaliças e prática de atividade física). As associações foram avaliadas por modelos logísticos, considerando-se o ajuste por potenciais fatores de confusão. Participaram da análise 5.827 indivíduos; $32,2 \%$ não saíram de casa na última semana; e entre os que saíram de casa, 97,5\% usaram máscara facial e 97,3\% higienizaram as mãos quando retornaram ao domicílio. A prática de atividade física nos niveis recomendados foi associada com menor chance de não sair de casa na semana anterior. Os exfumantes apresentaram maior chance de usar máscara e aqueles que praticavam atividade física tinham menor chance de adotar esta medida protetiva. Indivíduos com consumo de baixo risco de bebidas alcoólicas apresentaram maior chance de higienizar as mãos. Ações que visem ao aumento da adoção das medidas protetivas para o combate ao novo coronavírus devem considerar a existência de grupos vulneráveis, que podem ser identificados pela distribuição de outros comportamentos em saúde na população.

Infecções por Coronavírus; Inquéritos Epidemiológicos; Entrevistas por Telefone; Fatores Epidemiológicos; Comportamento Relacionado com a Saúde

\author{
Correspondência \\ S. V. Peixoto \\ Instituto René Rachou, Fundação Oswaldo Cruz. \\ Av. Augusto de Lima 1715, Belo Horizonte, MG 30190-009, \\ Brasil. \\ sergio.peixoto@fiocruz.br \\ 1 Instituto René Rachou, Fundação Oswaldo Cruz, \\ Belo Horizonte, Brasil. \\ 2 Escola de Enfermagem, Universidade Federal de Minas Gerais, \\ Belo Horizonte, Brasil. \\ 3 Programa de Pós-graduação em Saúde Púbica, Universidade \\ Federal de Minas Gerais, Belo Horizonte, Brasil.
}




\section{Introdução}

A pandemia da COVID-19, doença respiratória causada pelo novo coronavírus (SARS-CoV-2), constitui uma importante emergência em saúde pública. A rápida expansão de uma doença de elevada transmissibilidade, aliada à ausência de uma vacina ou tratamento efetivo para esta condição, exige a adoção de medidas não farmacológicas, que visam à redução da velocidade de transmissão do vírus, evitando o colapso dos sistemas de saúde. Medidas de distanciamento social, isolamento de casos, fechamento de escolas e comércio, restrição de tráfego e uso de máscaras faciais estão entre as estratégias mais adotadas em diferentes países 1,2,3,4.

A adesão às medidas de proteção individual é de extrema relevância para controlar a rápida disseminação do vírus 1,5. Estudos anteriores mostraram ampla variação na adoção dessas medidas em diferentes populações, durante a pandemia da COVID-19. O uso de máscaras em locais públicos foi reportado por 23,6\% dos adultos nos Estados Unidos 6, 63,2\% na Coreia do Sul 7 e por cerca de $98 \%$ na China 8 e em Hong Kong (SAR China) ${ }^{9}$. Já o hábito de higienizar as mãos frequentemente foi relatado por 58,5\% dos entrevistados em Tóquio, Japão 10, 67,8\% na Coreia do Sul 7 e 92,3\% em Hong Kong 9. Em relação ao distanciamento social, 41,5\% dos adultos participantes de inquérito na Coreia do Sul reportaram evitar locais com aglomeração de pessoas 7, enquanto na China este percentual atingiu mais de $95 \%$ 8,11. No Brasil, um inquérito on-line com cerca de 45 mil participantes, conduzido entre 25 de abril e 25 de maio de 2020 , identificou $75 \%$ de adesão ao distanciamento social 12 .

No geral, os comportamentos preventivos individuais frente às epidemias podem ser influenciados por fatores psicossociais, demográficos e comportamentos em saúde 7,11,13,14,15,16. O uso de máscaras em locais públicos e o hábito de lavar as mãos frequentemente, por exemplo, foram mais comuns entre mulheres, indivíduos mais velhos, com maior nível socioeconômico, com menores níveis de estresse, ansiedade e depressão e entre aqueles com maior percepção sobre a severidade da doença 6,7,11,13,15. Por outro lado, as associações entre a adoção de medidas protetivas e variáveis relacionadas aos comportamentos em saúde são menos exploradas na literatura. Estudos recentes não evidenciaram associações entre a adoção dessas medidas e o tabagismo e o consumo de bebidas alcoólicas 10,14. No entanto, pelo nosso conhecimento, essas associações ainda não foram exploradas em países da América Latina.

Os comportamentos em saúde tendem a se agrupar nas populações. A adoção de um comportamento saudável aumenta a chance de adesão a outros comportamentos protetivos, como ocorre para os principais fatores de risco para doenças crônicas não transmissíveis 17,18. Portanto, a hipótese a ser avaliada no presente artigo é que indivíduos com comportamentos saudáveis, como não fumar, não apresentar consumo de risco para bebidas alcoólicas, consumir frutas e hortaliças regularmente e praticar atividades físicas nos níveis recomendados tendem a aderir com mais frequência às medidas de proteção individual durante a pandemia da COVID-19.

Dessa forma, o presente trabalho tem por objetivo avaliar se os comportamentos saudáveis determinam a adoção de medidas protetivas individuais (não sair de casa, usar máscara em locais públicos e lavar as mãos) para combater a COVID-19, entre brasileiros com 50 anos ou mais de idade.

\section{Metodologia}

\section{Delineamento do ELSI-Brasil}

O Estudo Longitudinal da Saúde dos Idosos Brasileiros (ELSI-Brasil) é uma pesquisa de base domiciliar sobre as condições de vida e de saúde de adultos mais velhos, conduzida em amostra nacional representativa da população brasileira com 50 anos ou mais de idade. Para a composição da amostra, os municípios foram alocados em quatro estratos, de acordo com o tamanho da população residente. Nos três primeiros estratos, constituídos por municípios com até 750 mil habitantes, a amostra foi selecionada em três estágios (município, setor censitário e domicílio). No quarto estrato, composto com os municípios de grande porte, a seleção da amostra foi realizada em dois estágios (setor censitário e domicílio), incluindo todos os municípios desta categoria. Considerando essa estratégia amostral, estimou-se a participação de 10 mil indivíduos, na faixa etária considerada, residentes em 70 municí- 
pios das diferentes regiões do país. Maiores detalhes podem ser vistos na homepage da pesquisa (http:// elsi.cpqrr.fiocruz.br/) e em publicação anterior 19. O ELSI-Brasil foi aprovado pelo Comitê de Ética em Pesquisa da Fundação Oswaldo Cruz (Fiocruz), Minas Gerais (CAAE: 34649814.3.0000.5091) e todos os participantes assinaram o Termo de Consentimento Livre e Esclarecido.

A linha de base do estudo foi realizada em 2015 e 2016, tendo sido entrevistados 9.412 brasileiros com 50 anos ou mais. A segunda onda da pesquisa iniciou em agosto de 2019, mas foi interrompida em 17 de março de 2020 devido à pandemia da COVID-19. Até essa data, 9.177 indivíduos haviam sido entrevistados e examinados. Nesse momento, os participantes informaram seus números de telefones (fixos e/ou móveis), bem como aqueles de seus principais contatos pessoais, de modo a permitir a localização dos participantes em momentos futuros.

\section{Delineamento do inquérito telefônico (iniciativa ELSI-COVID-19)}

O ELSI-COVID-19 é uma iniciativa planejada para produzir informações sobre a epidemia do novo Coronavírus entre adultos brasileiros mais velhos, por meio de entrevistas telefônicas com os participantes da segunda onda do ELSI-Brasil. Todos os 9.177 participantes entrevistados até a interrupção da coleta de dados, em 17 de março de 2020, foram elegíveis para esta pesquisa. A entrevista telefônica durava cerca de cinco minutos e incluiu informações sobre medidas de prevenção, obtenção de medicamentos, diagnóstico para a COVID-19, uso de serviços de saúde e aspectos da saúde mental, sendo realizada por entrevistadores previamente treinados. A coleta de dados foi conduzida entre os dias 26 de maio e 08 de junho de 2020. Entre os 9.177 participantes da segunda onda do ELSI-Brasil, 6.149 (67\%) participaram da entrevista telefônica. Maiores detalhes podem ser obtidos em outra publicação 20.

\section{Variáveis}

Os desfechos do presente trabalho foram três medidas protetivas individuais, adotadas pela população frente à pandemia da COVID-19, coletadas na iniciativa ELSI-COVID-19. As medidas consideradas foram: (a) não ter saído de casa (como indicador do distanciamento social); (b) usar máscara quando saiu de casa; e (c) higienizar as mãos quando voltou para casa, tendo sempre a última semana como referência. O primeiro desfecho foi avaliado pela resposta "não saiu de casa" à pergunta: "Na última semana, quantos dias o(a) $\mathrm{Sr}($ a) saiu de casa?"; o uso de máscara foi avaliado pela resposta "sempre” à pergunta: “Na última semana, quando saiu de casa, o(a) Sr(a) usou máscara?”; e a higienização das mãos foi avaliada pela resposta "sempre" à questão: "Na última semana, quando saiu de casa, o(a) $\mathrm{Sr}(a)$ lavou as mãos ou usou álcool em gel quando voltou para casa?". As duas últimas perguntas foram respondidas apenas por aqueles que relataram ter saído de casa na semana anterior à entrevista.

As variáveis explicativas, coletadas na segunda onda do ELSI-Brasil, foram os seguintes comportamentos em saúde: tabagismo (fumante atual, ex-fumante, não fumante), consumo de bebidas alcoólicas (consumo de risco, consumo de baixo risco e não consumo), consumo de frutas e hortaliças em níveis recomendados (não e sim) e prática de atividade física nos níveis recomendados (não e sim).

Foram considerados fumantes atuais os entrevistados que reportaram fumar 100 ou mais cigarros ao longo da vida e continuavam fumando no momento da entrevista, independentemente da frequência. Os ex-fumantes foram aqueles que reportaram já ter fumado 100 ou mais cigarros ao longo da vida, mas que haviam interrompido o hábito e não fumavam no momento da entrevista. Os demais foram considerados não fumantes. O consumo de bebidas alcoólicas foi avaliado pela frequência de consumo e número de doses por dia. Aqueles participantes que relataram não consumir ou consumir bebidas alcoólicas menos de uma vez por semana foram classificados como não consumo. Entre os demais, considerou-se como baixo risco o consumo semanal menor ou igual a sete doses para mulheres e 14 doses para homens, já o consumo de risco foi considerado quando houve relato de consumo diário superior a sete doses semanais para mulheres e 14 doses semanais para homens, conforme recomendações do Instituto Nacional de Abuso de Álcool e Alcoolismo (National Institute on Alcohol Abuse and Alcoholism) 21. Além disso, os participantes que reportaram consumo binge, ou seja, quatro ou mais doses/dia para mulheres e cinco ou mais doses/dia para homens, em uma única ocasião, foram classificados como consumo de risco. 
O consumo recomendado de frutas e hortaliças considerou as diretrizes da Organização Mundial da Saúde (OMS) e da Organização das Nações Unidas para a Alimentação e a Agricultura (FAO) 22. Para estimar esse consumo foram consideradas a frequência semanal e diária de frutas, suco de frutas natural e hortaliças. Aqueles indivíduos que reportaram consumo de frutas ou sucos e de hortaliças em cinco ou mais dias da semana e que relataram o consumo diário maior ou igual a cinco porções destes grupos de alimentos, devendo conter frutas ou sucos e hortaliças todos os dias, foram considerados como tendo o consumo recomendado.

O nível de atividade física foi avaliado pelo questionário IPAQ (International Physical Activity Questionnaire), versão reduzida, que foi traduzida e validada para o Brasil 23. Foi considerada a frequência (dias por semana) e duração (tempo por dia) das atividades físicas realizadas, por pelo menos 10 minutos contínuos, na semana anterior à entrevista. As atividades incluídas foram: (a) caminhada (em casa ou no trabalho, como forma de transporte para ir de um lugar para outro, por lazer, por prazer ou como forma de exercício); (b) atividades moderadas (como pedalar leve na bicicleta, nadar, dançar, fazer ginástica aeróbica leve, jogar vôlei recreativo, carregar pesos leves, fazer serviços domésticos na casa, no quintal ou no jardim como varrer, aspirar, cuidar do jardim etc., mas não incluindo caminhada); e (c) atividades vigorosas (como correr, fazer ginástica aeróbica, jogar futebol, pedalar rápido na bicicleta, jogar basquete, fazer serviços domésticos pesados em casa, no quintal ou cavoucar no jardim, carregar pesos etc.). Com base nessas informações foi estimado o tempo total de prática de atividade física, considerando-se o tempo gasto em atividades vigorosas em dobro. Conforme recomendações da OMS 24, os indivíduos que praticaram atividade física em 150 minutos ou mais por semana foram classificados como tendo níveis recomendados da prática.

Os potenciais fatores de confusão foram selecionados conforme a literatura recente 6,7,10,11,14,16, sendo todos coletados na segunda onda do ELSI-Brasil, incluindo: sexo (feminino, masculino), faixa etária (em anos), escolaridade em anos de estudos (<4, 4-7 e 8 ou mais), estado conjugal (não casado, casado/união estável), cor da pele (branca, não branca), número de moradores no domicílio (1, 2, 3 ou mais) e número de condições crônicas autorreferidas (nenhuma, 1, 2 ou mais), incluindo hipertensão, diabetes, infarto, insuficiência cardíaca, angina, depressão, artrite, câncer, insuficiência renal e asma. Além desses, a região de residência (Norte, Nordeste, Sudeste, Sul e Centro-oeste) foi também incluída nesta análise.

\section{Análise dos dados}

A caracterização da amostra estudada foi realizada para todas as variáveis incluídas no estudo pela distribuição proporcional das mesmas, considerando-se a amostra total. A distribuição dessas variáveis segundo os desfechos estudados pode ser vista em material suplementar (http://cadernos.ensp. fiocruz.br/static/arquivo/csp-1954-20-material-suplementar-port_1681.pdf).

As associações entre os comportamentos em saúde, avaliados na segunda onda do ELSI-Brasil, e as medidas protetivas individuais para o combate ao novo coronavírus, reportadas na iniciativa ELSI-COVID-19, foram avaliadas por modelos logísticos de regressão, estimando-se odds ratio (OR) e os respectivos intervalos de 95\% de confiança (IC95\%). Foram construídos dois modelos de regressão para cada desfecho, sendo um modelo bruto e outro ajustado por todos os fatores de confusão incluídos nesta análise. Foram consideradas significativas as associações com valor de $\mathrm{p}<5 \%$.

Todas as análises foram realizadas no Stata, versão 14.0 (https://www.stata.com), considerando-se o peso e o efeito do delineamento amostral. Os pesos foram estimados especificamente para aqueles que responderam à entrevista telefônica, considerando-se o estrato natural, a idade, o sexo e o nível de escolaridade 20 .

\section{Resultados}

A presente análise incluiu 5.827 adultos mais velhos, participantes da iniciativa ELSI-COVID-19 que possuíam todas as informações consideradas. Com relação às prevalências dos desfechos avaliados, os resultados deste trabalho mostraram que 32,2\% (IC95\%: 29,1-35,4) dos entrevistados não saíram de casa na última semana. Entre aqueles que saíram de casa $(\mathrm{n}=3.435), 97,5 \%($ IC95\%: 96,6-98,2) 
reportaram sempre usar máscara facial e 97,3\% (IC95\%: 95,7-98,4) reportaram higienizar as mãos quando retornaram à casa.

A Tabela 1 apresenta as características da amostra para a população total. A amostra analisada teve predominância de mulheres (54,8\%), indivíduos com idades entre 50 e 59 anos (46,9\%), com oito anos ou mais de escolaridade (56,1\%), casados (60,3\%), cor da pele branca $(52,5 \%)$, residentes em domićlios com dois moradores $(39,6 \%)$, residentes na Região Sudeste $(40,6 \%)$ e com nenhuma condição crônica (34,7\%). Além disso, a maioria dos participantes não fumava (68\%), não consumia bebida alcoólica $(78,2 \%)$, não tinha consumo recomendado de frutas e hortaliças $(88,3 \%)$ e atingia o nível de atividade física semanal recomendado (52,9\%).

A Tabela 2 mostra a distribuição dos comportamentos em saúde segundo o desfecho não sair de casa na última semana, e descreve a magnitude das associações entre os comportamentos em saúde e este desfecho. Após ajuste pelos fatores de confusão considerados, os praticantes de atividades físicas nos níveis recomendados tinham menor chance de não terem saído de casa na semana anterior $(\mathrm{OR}=$ 0,69; IC95\%: 0,53-0,91).

\section{Tabela 1}

Distribuição das características dos participantes do estudo na amostra total. Iniciativa ELSI-COVID-19, 2020.

\begin{tabular}{|c|c|}
\hline Variáveis & $\%(I C 95 \%)$ \\
\hline \multicolumn{2}{|l|}{ Sexo } \\
\hline Feminino & $54,8(51,3-58,3)$ \\
\hline Masculino & $45,2(41,7-48,7)$ \\
\hline \multicolumn{2}{|l|}{ Faixa etária (anos) } \\
\hline $50-59$ & $46,9(41,9-52,1)$ \\
\hline $60-69$ & $29,3(27,2-31,6)$ \\
\hline $70-79$ & $16,3(13,3-19,7)$ \\
\hline 80 ou mais & $7,5(5,9-9,5)$ \\
\hline \multicolumn{2}{|c|}{ Escolaridade (anos de estudos) } \\
\hline$<4$ & $17,4(14,7-20,6)$ \\
\hline $4-7$ & $26,5(23,8-29,4)$ \\
\hline 8 ou mais & $56,1(51,4-60,7)$ \\
\hline \multicolumn{2}{|l|}{ Estado conjugal } \\
\hline Não casado & $39,7(35,5-43,9)$ \\
\hline Casado/União estável & $60,3(56,1-64,5)$ \\
\hline \multicolumn{2}{|l|}{ Cor da pele } \\
\hline Branca & $52,5(44,9-60,0)$ \\
\hline Não branca & $47,5(40,0-55,1)$ \\
\hline \multicolumn{2}{|c|}{ Número de moradores no domicilio } \\
\hline 1 & $23,0(18,9-27,6)$ \\
\hline 2 & $39,6(36,5-42,9)$ \\
\hline 3 ou mais & $37,4(32,1-43,0)$ \\
\hline \multicolumn{2}{|l|}{ Região de residência } \\
\hline Norte & $6,3(1,8-19,1)$ \\
\hline Nordeste & $26,8(17,2-39,3)$ \\
\hline Sudeste & $40,6(27,9-54,8)$ \\
\hline Sul & $15,7(7,5-29,9)$ \\
\hline Centro-oeste & $10,6(4,9-21,4)$ \\
\hline \multicolumn{2}{|c|}{ Número de condições crônicas } \\
\hline Nenhuma & $34,7(31,0-38,6)$ \\
\hline 1 & $32,9(30,6-35,3)$ \\
\hline 2 ou mais & $32,4(28,6-36,5)$ \\
\hline
\end{tabular}

(continua) 
Tabela 1 (continuação)

\begin{tabular}{lc}
\hline Variáveis & $\%(\mathbf{I C 9 5 \% )}$ \\
\hline Tabagismo & $11,2(9,6-12,9)$ \\
$\quad$ Fumante atual & $20,8(17,6-24,5)$ \\
$\quad$ Ex-fumante & $68,0(63,9-71,8)$ \\
$\quad$ Não fumante & \\
Consumo de bebidas alcoólicas * & $12,6(9,9-15,9)$ \\
$\quad$ Consumo de risco & $9,2(6,9-12,1)$ \\
$\quad$ Consumo de baixo risco & $78,2(74,6-81,4)$ \\
$\quad$ Não consumo & \\
Consumo recomendado de frutas e hortaliças ** & $88,3(85,3-90,8)$ \\
$\quad$ Não & $11,7(9,2-14,7)$ \\
Sim & \\
Atividade física nos níveis recomendados *** & $47,1(42,2-51,9)$ \\
Não & $52,9(48,1-57,8)$ \\
Sim &
\end{tabular}

IC95\%: intervalo de 95\% confiança.

* Consumo de baixo risco: $\leq 7$ doses/semana para mulheres ou $\leq 14$ doses/semana para homens; Consumo de risco: > 7 doses/semana para mulheres ou > 14 doses/semana para homens; inclui também aqueles que reportaram $\geq 4$ doses/ dia para mulheres ou $\geq 5$ doses/dia para homens, em uma única ocasião, nos últimos 30 dias;

** Consumo de 5 ou mais porções/dia em 5 ou mais dias/semana;

*** Mais de 150 minutos/semana, incluindo caminhada e atividades de intensidade moderada ou vigorosa.

\section{Tabela 2}

Associação entre os comportamentos em saúde e o desfecho não ter saído de casa na última semana. Iniciativa ELSI-COVID-19, 2020.

\begin{tabular}{|c|c|c|c|c|}
\hline \multirow[t]{2}{*}{ Comportamentos em saúde } & \multicolumn{2}{|c|}{$\begin{array}{c}\text { Saiu de casa na última } \\
\text { semana (\%) }\end{array}$} & \multicolumn{2}{|c|}{ OR (IC95\%) } \\
\hline & Sim & Não & Modelo bruto & Modelo ajustado \\
\hline \multicolumn{5}{|l|}{ Tabagismo } \\
\hline Fumante atual & 11,0 & 11,3 & 1,00 & 1,00 \\
\hline Ex-fumante & 21,1 & 20,4 & $0,94(0,68-1,32)$ & $0,77(0,55-1,03)$ \\
\hline Não fumante & 67,9 & 68,3 & $0,98(0,70-1,36)$ & $0,72(0,51-1,02)$ \\
\hline \multicolumn{5}{|l|}{ Consumo de bebidas alcoólicas * } \\
\hline Consumo de risco & 15,0 & 7,5 & 1,00 & 1,00 \\
\hline Consumo de baixo risco & 11,6 & 4,2 & $0,72(0,36-1,42)$ & $0,76(0,37-1,56)$ \\
\hline Não consumo & 73,4 & 88,3 & $2,39(1,57-3,65)$ & $1,61(0,98-2,64)$ \\
\hline \multicolumn{5}{|c|}{$\begin{array}{l}\text { Consumo recomendado de frutas e } \\
\text { hortaliças ** }\end{array}$} \\
\hline Não & 88,7 & 87,5 & 1,00 & 1,00 \\
\hline Sim & 11,3 & 12,5 & $1,12(0,76-1,65)$ & $1,25(0,84-1,85)$ \\
\hline \multicolumn{5}{|l|}{$\begin{array}{l}\text { Atividade física nos níveis } \\
\text { recomendados } * * \star\end{array}$} \\
\hline Não & 42,2 & 57,2 & 1,00 & 1,00 \\
\hline Sim & 57,8 & 42,8 & $0,55(0,43-0,69)$ & $0,69(0,53-0,91)$ \\
\hline
\end{tabular}

IC95\%: intervalo de 95\% de confiança; OR: odds ratio.

Nota: OR e IC95\% obtidos pela regressão logística. Ajustado pelas variáveis listadas na tabela, além de sexo, faixa etária, escolaridade, estado conjugal, cor da pele, número de moradores no domicílio, região de residência e número de doenças crônicas, assim como descritas na Tabela 1.

* Consumo de baixo risco: $\leq 7$ doses/semana para mulheres ou $\leq 14$ doses/semana para homens; Consumo de risco: > 7 doses/semana para mulheres ou $>14$ doses/semana para homens; inclui também aqueles que reportaram $\geq 4$ doses/ dia para mulheres ou $\geq 5$ doses/dia para homens, em uma única ocasião, nos últimos 30 dias;

** Consumo de 5 ou mais porções/dia em 5 ou mais dias/semana;

*** Mais de 150 minutos/semana, incluindo caminhada e atividades de intensidade moderada ou vigorosa. 
A Tabela 3 apresenta a distribuição dos comportamentos em saúde segundo o uso de máscara facial e descreve a magnitude das associações entre estes comportamentos e o uso de máscara, entre aqueles que saíram de casa na última semana. Os ex-fumantes registraram maior chance de usar máscara em público (OR = 1,89; IC95\%: 1,05-3,42), quando comparados àqueles que eram fumantes atuais. Por outro lado, os praticantes de atividade física nos níveis recomendados tiveram menor chance de adotar essa medida protetiva (OR = 0,51; IC95\%: 0,31-0,86).

A Tabela 4 mostra a distribuição dos comportamentos em saúde segundo a higienização das mãos entre aqueles que saíram de casa na última semana, e descreve a magnitude das associações entre os comportamentos em saúde e este desfecho. Somente aqueles participantes que foram classificados como tendo um consumo de bebidas alcoólicas de baixo risco apresentaram maior chance de adotar essa medida de proteção individual (OR = 4,24; IC95\%: 1,08-16,64), quando comparados aos que apresentaram consumo de risco.

\section{Tabela 3}

Associação entre os comportamentos em saúde e o uso de máscara facial dentre aqueles que saíram de casa na última semana. Iniciativa ELSI-COVID-19, 2020.

\begin{tabular}{|c|c|c|c|c|}
\hline \multirow[t]{2}{*}{ Comportamentos em saúde } & \multicolumn{2}{|c|}{ Uso de máscara facial (\%) } & \multicolumn{2}{|c|}{ OR (IC95\%) } \\
\hline & Não & Sim & Modelo bruto & $\begin{array}{l}\text { Modelo } \\
\text { ajustado }\end{array}$ \\
\hline \multicolumn{5}{|l|}{ Tabagismo } \\
\hline Fumante atual & 15,7 & 10,9 & 1,00 & 1,00 \\
\hline Ex-fumante & 16,6 & 21,2 & $1,83(1,07-3,13)$ & $1,89(1,05-3,42)$ \\
\hline Não fumante & 67,7 & 67,9 & $1,44(0,76-2,73)$ & $1,12(0,58-2,17)$ \\
\hline \multicolumn{5}{|l|}{ Consumo de bebidas alcoólicas * } \\
\hline Consumo de risco & 17,4 & 15,0 & 1,00 & 1,00 \\
\hline Consumo de baixo risco & 13,1 & 11,6 & $1,03(0,17-6,37)$ & $0,59(0,09-4,03)$ \\
\hline Não consumo & 69,4 & 73,4 & $1,23(0,61-2,51)$ & $0,94(0,45-1,95)$ \\
\hline \multicolumn{5}{|c|}{ Consumo recomendado de frutas e } \\
\hline \multicolumn{5}{|c|}{ hortaliças ** } \\
\hline Não & 97,3 & 88,5 & 1,00 & 1,00 \\
\hline Sim & 2,7 & 11,5 & $4,67(1,50-14,59)$ & $3,29(0,91-11,89)$ \\
\hline \multirow{2}{*}{\multicolumn{5}{|c|}{$\begin{array}{l}\text { Atividade física nos níveis } \\
\text { recomendados *** }\end{array}$}} \\
\hline & & & & \\
\hline Não & 33,0 & 42,5 & 1,00 & 1,00 \\
\hline Sim & 67,0 & 57,5 & $0,66(0,36-1,21)$ & $0,51(0,31-0,86)$ \\
\hline
\end{tabular}

IC95\%: intervalo de 95\% de confiança; OR: odds ratio.

Nota: OR e IC95\% obtidos pela regressão logística. Ajustado pelas variáveis listadas na tabela, além de sexo, faixa etária, escolaridade, estado conjugal, cor da pele, número de moradores no domicílio, região de residência e número de doenças crônicas, assim como descritas na Tabela 1.

* Consumo de baixo risco: $\leq 7$ doses/semana para mulheres ou $\leq 14$ doses/semana para homens; Consumo de risco: >

7 doses/semana para mulheres ou $>14$ doses/semana para homens; inclui também aqueles que reportaram $\geq 4$ doses/ dia para mulheres ou $\geq 5$ doses/dia para homens, em uma única ocasião, nos últimos 30 dias;

** Consumo de 5 ou mais porções/dia em 5 ou mais dias/semana;

*** Mais de 150 minutos/semana, incluindo caminhada e atividades de intensidade moderada ou vigorosa. 
Tabela 4

Associação entre os comportamentos em saúde e a higienização das mãos dentre aqueles que saíram de casa na última semana. Iniciativa ELSI-COVID-19, 2020.

\begin{tabular}{|c|c|c|c|c|}
\hline \multirow[t]{2}{*}{ Comportamentos em saúde } & \multicolumn{2}{|c|}{ Higienização das mãos (\%) } & \multicolumn{2}{|c|}{ OR (IC95\%) } \\
\hline & Não & Sim & Modelo bruto & Modelo ajustado \\
\hline \multicolumn{5}{|l|}{ Tabagismo } \\
\hline Fumante atual & 24,5 & 10,7 & 1,00 & 1,00 \\
\hline Ex-fumante & 22,9 & 21,0 & $2,11(0,50-8,88)$ & $1,82(0,56-5,87)$ \\
\hline Não fumante & 52,6 & 68,3 & $2,98(0,77-11,59)$ & $2,26(0,89-5,73)$ \\
\hline \multicolumn{5}{|l|}{ Consumo de bebidas alcoólicas * } \\
\hline Consumo de risco & 33,8 & 14,5 & 1,00 & 1,00 \\
\hline Consumo de baixo risco & 4,7 & 11,8 & $5,43(1,11-26,52)$ & $4,24(1,08-16,64)$ \\
\hline Não consumo & 63,4 & 73,7 & $2,54(0,82-7,91)$ & $1,83(0,80-4,18)$ \\
\hline \multirow{2}{*}{\multicolumn{5}{|c|}{$\begin{array}{l}\text { Consumo recomendado de frutas e } \\
\text { hortaliças ** }\end{array}$}} \\
\hline & & & & \\
\hline Não & 90,4 & 88,6 & 1,00 & 1,00 \\
\hline Sim & 9,6 & 11,4 & $1,21(0,40-3,62)$ & $0,89(0,29-2,76)$ \\
\hline \multirow{2}{*}{\multicolumn{5}{|c|}{$\begin{array}{l}\text { Atividade física nos níveis } \\
\text { recomendados } \star \star \star\end{array}$}} \\
\hline & & & & \\
\hline Não & 38,1 & 42,4 & 1,00 & 1,00 \\
\hline Sim & 61,9 & 57,6 & $0,84(0,38-1,82)$ & $0,72(0,35-1,47)$ \\
\hline
\end{tabular}

IC95\%: intervalo de 95\% de confiança; OR: odds ratio.

Nota: OR e IC95\% obtidos pela regressão logística. Ajustado pelas variáveis listadas na tabela, além de sexo, faixa etária, escolaridade, estado conjugal, cor da pele, número de moradores no domicílio, região de residência e número de doenças crônicas assim como descritas na Tabela 1.

* Consumo de baixo risco: $\leq 7$ doses/semana para mulheres ou $\leq 14$ doses/semana para homens; Consumo de risco: > 7 doses/semana para mulheres ou > 14 doses/semana para homens; inclui também aqueles que reportaram $\geq 4$ doses/ dia para mulheres ou $\geq 5$ doses/dia para homens, em uma única ocasião, nos últimos 30 dias;

** Consumo de 5 ou mais porções/dia em 5 ou mais dias/semana;

*** Mais de 150 minutos/semana, incluindo caminhada e atividades de intensidade moderada ou vigorosa.

\section{Discussão}

Os resultados deste estudo evidenciaram que cerca de $1 / 3$ da população brasileira com 50 anos ou mais não saiu de casa na semana anterior à entrevista e, entre aqueles que saíram de casa, a quase totalidade reportou usar máscara (97,5\%) e higienizar as mãos ao retornar (97,3\%). Além disso, não sair de casa foi significativamente e negativamente associado à prática de atividade física nos níveis recomendados. O uso de máscara em público foi mais frequente entre os ex-fumantes, mas os praticantes de atividade física em níveis recomendados tiveram menor chance de adotar esta medida protetiva. Sobre o hábito de higienizar as mãos ao retornar ao domicílio, apenas o grupo com consumo de bebidas alcoólicas de baixo risco apresentou maior chance de adotar esta medida de proteção individual, comparado aos que apresentavam consumo de risco.

A adoção de práticas de distanciamento social, como evitar locais públicos ou com aglomeração de pessoas e permanecer em casa sempre que possível, apresenta grande variação entre os inquéritos conduzidos durante a pandemia do novo coronavírus 7,8,10,16,25. O percentual de adoção dessas práticas variou de 29,6\% em Tóquio 10 a 96,4\% na China 11 e essas diferenças podem ser atribuídas, sobretudo, à composição da amostra, que na maioria das vezes é intencional, ao período de realização e à forma de condução do inquérito ou à forma de se realizar a pergunta, incluindo o período de referência da mesma. Em Hong Kong e em Tóquio, por exemplo, o percentual da população que reportou evitar locais com aglomeração de pessoas sofreu aumento importante ao longo do período 
da pandemia do SARS-CoV-2 1,14. Esses aumentos podem, portanto, retratar a evolução da epidemia nesses locais, levando à recomendação de novas medidas de controle, como o distanciamento social.

No Brasil, dados do VIGITEL (Vigilância de Fatores de Risco e Proteção para Doenças Crônicas por Inquérito Telefônico) COVID-19 mostraram que 89,9\% da população adulta residente nas capitais e Distrito Federal reportavam isolamento social, considerado como ter evitado sair de casa a menos que fosse necessário, ter evitado aglomerações de pessoas ou lugares muito cheios e ter evitado contato próximo com outras pessoas, como cumprimentos ou abraços 26. Entretanto, outra pesquisa on-line, conduzida pela Fiocruz, Universidade Federal de Minas Gerais (UFMG) e Universidade Estadual de Campinas (Unicamp), com cerca de 45 mil respondentes, mostrou que a adesão ao isolamento social foi de $75 \%$, sendo que $60 \%$ referiram ter saído para compras de supermercado e farmácia e $15 \%$ ficaram rigorosamente em casa, só tendo saído para necessidades de saúde, como ir ao médico 12. Portanto, o menor percentual observado no presente estudo $(32,2 \%)$ pode ser atribuído à pergunta mais restritiva (não ter saído de casa na semana anterior à entrevista), indicando a parcela dos adultos mais velhos que adotaram o completo distanciamento físico. De toda forma, até o dia 13 de junho de 2020, o Brasil ocupava a segunda posição do mundo em número de casos (850.514) e óbitos (41.828) pela COVID-19 26, o que demonstra a necessidade de maior adesão às medidas de distanciamento físico. Evidências apontam que o distanciamento social é efetivo em reduzir a disseminação do vírus e retardar o aumento do número de casos 4,27,28, embora a sustentabilidade desta medida dependa da implementação de políticas de proteção social, o que deve ser considerado pelos governantes 29 .

Em relação ao uso de máscara em locais públicos e ao hábito de higienizar as mãos ao retornar para o domicílio, cerca de $97 \%$ da população brasileira com 50 anos ou mais reportaram adotar estes hábitos, de forma semelhante ao observado em populações de outras regiões, como China 8,30, Hong Kong 1,9, Paquistão 25 e Japão 16. As medidas de proteção individual são fundamentais para o controle da transmissão de doenças respiratórias, ainda que a efetividade do uso de máscaras pela população em geral seja questionada 31,32. Ainda assim, a adoção dessas medidas tem sido amplamente recomendada 32,33,34, podendo contribuir para a redução da transmissão do vírus, minimizando o tempo do isolamento social, que pode ter impacto negativo na economia e na saúde mental das populações 5,35.

No presente estudo, o relato de não ter saído de casa na última semana foi menos comum entre aqueles que praticavam atividade física nos níveis recomendados. A redução da prática da atividade física e o aumento do tempo sedentário, durante o distanciamento social devido à COVID-19, foram reportados para o Brasil em inquérito on-line. Entre os idosos, por exemplo, a prevalência da prática de atividade física reduziu de 30,4\% para 14,2\% 36. No entanto, um estudo conduzido no Canadá observou que indivíduos ativos reportavam maiores níveis de atividade física durante a pandemia, em comparação ao grupo dos inativos 37 . Essa evidência pode justificar a associação inversa observada no presente estudo, fazendo com que os indivíduos com os maiores níveis de atividade física antes da pandemia buscassem manter esta prática, realizando atividades fora do domicílio. Mesmo sabendo que a prática de atividade física pode minimizar os efeitos negativos do distanciamento social para saúde mental dos indivíduos 38 , as recomendações devem buscar estratégias para a manutenção desta prática no domicílio, como o uso de tecnologias digitais para orientar a população 39,40.

Os resultados deste estudo mostraram que o uso de máscara em locais públicos foi mais frequente entre os ex-fumantes, mas foi menos frequente entre os indivíduos que praticavam atividade física em níveis recomendados. Estudos anteriores não encontraram associação significativa entre a adoção de medidas de controle da COVID-19 e o hábito de fumar 10,14,16. No entanto, chama atenção que a interrupção do tabagismo pode estar relacionada à adoção de outros comportamentos saudáveis, como maior consumo de frutas e hortaliças e a prática de atividade física 17,18 , o que pode favorecer o entendimento da importância de outras medidas de proteção individual, como aquelas recomendadas para o combate ao novo coronavírus.

Em relação à menor chance de uso de máscara entre aqueles que praticavam atividade física nos níveis recomendados, sugere-se que esta medida de proteção individual possa dificultar a realização de exercícios físicos, causando desconforto para respirar. Alguns estudos já demonstraram relato de desconforto pelo uso de máscaras 41 , além de reações alérgicas na pele 42 , o que pode dificultar a adesão a esta medida protetiva. Além disso, a máscara pode causar resistência à inalação, tornando a respiração mais difícil com o aumento do tempo de uso 43, o que pode diminuir sua adesão entre os praticantes de atividade física. 
O hábito de lavar as mãos ao retornar ao domicílio foi mais comum entre os indivíduos que apresentavam consumo de baixo risco de bebidas alcoólicas, em comparação ao grupo com consumo de risco. Embora não tenha sido encontrado outro estudo semelhante no contexto da pandemia da COVID-19, ressalta-se que o consumo de baixo risco de bebidas alcoólicas tem sido associado com a melhor dieta e com o fato de o indivíduo não fumar 44,45, o que pode ser também coerente com a adoção de medidas de higiene pessoal, como o hábito de lavar as mãos, tal como foi observado neste trabalho.

A interpretação dos resultados apresentados neste estudo deve considerar a dificuldade nas comparações com outras pesquisas devido à escassez de investigações semelhantes, à diversidade da composição etária, às diferenças entre as perguntas usadas nos inquéritos e à variação na adoção de medidas de controle pelos diferentes países e regiões ao longo do tempo. Além disso, devem ser consideradas as dificuldades relacionadas à entrevista telefônica, como o não atendimento, a recusa em prestar informações por este meio e a existência de números de telefones não válidos ou não informados na segunda onda do ELSI-Brasil, embora o uso de pesos calibrados tenha minimizado esta limitação. A avaliação do uso de máscara e da higienização das mãos pode ter sido influenciada por viés de informação, pois os indivíduos poderiam tender a relatar o comportamento socialmente aceitável, superestimando a adoção destas medidas, o que pode ter diluído as forças de associação reportadas.

Por outro lado, a iniciativa ELSI-COVID-19 constitui uma excelente oportunidade para explorar aspectos relacionados à COVID-19 em amostra representativa da população brasileira com 50 anos ou mais de idade, proveniente das diferentes regiões geográficas do país. Ademais, as exposições avaliadas no presente trabalho foram coletadas em período imediatamente anterior ao início da epidemia no Brasil, possibilitando avaliar as relações temporais entre as exposições e os desfechos investigados.

Concluindo, os resultados evidenciaram que comportamentos em saúde, como tabagismo, consumo de bebidas alcoólicas e prática de atividade física, podem predizer a adoção de medidas protetivas individuais frente à pandemia da COVID-19, possibilitando a identificação de grupos com maior e menor chances de aderir a estas medidas. No entanto, a prática de atividade física em níveis recomendados tende a dificultar a permanência em casa e o uso de máscara em locais públicos, prejudicando o controle da transmissão do vírus nesse grupo. Nesse sentido, estratégias de comunicação para a orientação adequada aos indivíduos fisicamente ativos devem ser pensadas. Por fim, os resultados deste estudo podem contribuir com o planejamento de ações que visem ao aumento da adoção das medidas protetivas individuais pela população, utilizando os modelos das ciências sociais e do comportamento, considerando que esta adoção não é determinada apenas por escolhas individuais, mas podem depender de outros comportamentos em saúde e do contexto em que os indivíduos vivem 46. 


\section{Colaboradores}

S. V. Peixoto e M. A. Nascimento-Souza contribuíram na concepção do artigo, análise dos dados e redação do trabalho. J. V. M. Mambrini contribuiu na análise e interpretação dos dados e redação do trabalho. F. B. Andrade e M. F. Lima-Costa contribuíram no delineamento do estudo e revisão do manuscrito. D. C. Malta contribuiu na interpretação dos dados, redação e revisão do manuscrito.

\section{Informações adicionais}

ORCID: Sérgio Viana Peixoto (0000-0001-94312280); Mary Anne Nascimento-Souza (00000002-3525-6554); Juliana Vaz de Melo Mambrini (0000-0002-0420-3062); Fabiola Bof de Andrade (0000-0002-3467-3989); Deborah Carvalho Malta (0000-0002-8214-5734); Maria Fernanda LimaCosta (0000-0002-3474-2980).

\section{Agradecimentos}

O ELSI-Brasil foi financiado pelo Ministério da Saúde, Departamento de Ciência e Tecnologia da Secretaria de Ciência e Tecnologia e Insumos Estratégicos - DECIT/SCTI (404965/2012-1 e 28/2017) e Coordenação da Saúde da Pessoa Idosa - COSAPI/ DAPES/SAS (20836, 22566, 23700 e 77/2019). A iniciativa ELSI-COVID-19 é financiada pelo DECIT/SCTI e pelo Conselho Nacional de Desenvolvimento Científico e Tecnológico (CNPq). S. V. Peixoto, D. C. Malta e M. F. Lima-Costa são bolsistas de produtividade em pesquisa do CNPq.

\section{Referências}

1. Cowling BJ, Ali ST, Ng TWY, Tsang TK, Li JCM, Fong MW, et al. Impact assessment of non-pharmaceutical interventions against coronavirus disease 2019 and influenza in Hong Kong: an observational study. Lancet Public Health 2020; 5:e279-88.

2. Ferguson N, Laydon D, Nedjati-Gilani G, Imai N, Ainslie K, Baguelin M, et al. Report 9 - Impact of non-pharmaceutical interventions (NPIs) to reduce COVID-19 mortality and healthcare demand, 2020. https://www. imperial.ac.uk/mrc-global-infectious-diseaseanalysis/covid-19/report-9-impact-of-npison-covid-19/ (acessado em 30/Jun/2020).

3. Ngonghala CN, Iboi E, Eikenberry S, Scotch $\mathrm{M}$, MacIntyre CR, Bonds $\mathrm{MH}$, et al. Mathematical assessment of the impact of nonpharmaceutical interventions on curtailing the 2019 novel Coronavirus. Math Biosci 2020; 325:108364.

4. Delen D, Eryarsoy E, Davazdahemami B. No place like home: cross-national data analysis of the efficacy of social distancing during the COVID-19 pandemic. JMIR Public Heal Surveill 2020; 6:e19862.
5. West R, Michie S, Rubin GJ, Amlôt R. Applying principles of behaviour change to reduce SARS-CoV-2 transmission. Nat Hum Behav 2020; 4:451-9.

6. Clements JM. Knowledge and behaviors toward COVID-19 among us residents during the early days of the pandemic: cross-sectional online questionnaire. JMIR Public Health Surveill 2020; 6:e19161.

7. Lee M, You M. Psychological and behavioral responses in South Korea during the early stages of coronavirus disease 2019 (COVID-19). Int J Environ Res Public Health 2020; 17:2977.

8. Huang Y, Wu Q, Wang P, Xu Y, Wang L, Zhao $\mathrm{Y}$, et al. Measures undertaken in China to avoid COVID-19 infection: internet-based, cross-sectional survey study. J Med Internet Res 2020; 22:e18718.

9. Chan EYY, Huang Z, Lo ESK, Hung KKC, Wong ELY, Wong SYS. Sociodemographic predictors of health risk perception, attitude and behavior practices associated with healthemergency disaster risk management for biological hazards: the case of COVID-19 pandemic in Hong Kong, SAR China. Int J Environ Res Public Health 2020; 17:3869. 
10. Machida M, Nakamura I, Saito R, Nakaya T, Hanibuchi T, Takamiya T, et al. Adoption of personal protective measures by ordinary citizens during the COVID-19 outbreak in Japan. Int J Infect Dis 2020; 94:139-44.

11. Zhong BL, Luo W, Li HM, Zhang QQ, Liu XG, Li WT, et al. Knowledge, attitudes, and practices towards COVID-19 among chinese residents during the rapid rise period of the COVID-19 outbreak: A quick online cross-sectional survey. Int J Biol Sci 2020; 16:1745-52.

12. Szwarcwald CL, Souza-Júnior PRB, Malta, DC, Barros MBA, Magalhães MAFM, Xavier DR. Adesão às medidas de restrição social e efeitos sobre a disseminação dos casos de COVID-19 no Brasil. Epidemiol Serv Saúde 2020; no prelo.

13. Bish A, Michie S. Demographic and attitudinal determinants of protective behaviours during a pandemic: a review. Br J Health Psychol 2010; 15:797-824.

14. Machida M, Nakamura I, Saito R, Nakaya T, Hanibuchi T, Takamjya T, et al. Changes in implementation of personal protective measures by ordinary japanese citizens: a longitudinal study from the early phase to the community transmission phase of the COVID-19 outbreak. Int J Infect Dis 2020; 96:371-5.

15. Wang C, Pan R, Wan X, Tan Y, Xu L, Ho CS, et al. Immediate psychological responses and associated factors during the initial stage of the 2019 coronavirus disease (COVID-19) epidemic among the general population in China. Int J Environ Res Public Health 2020; 17:1729.

16. Muto K, Yamamoto I, Nagasu M, Tanaka M, Wada K. Japanese citizens' behavioral changes and preparedness against COVID-19: an online survey during the early phase of the pandemic. PLoS One 2020; 15:e0234292.

17. Poortinga $\mathrm{W}$. The prevalence and clustering of four major lifestyle risk factors in an English adult population. Prev Med (Baltim) 2007; 44:124-8.

18. Geller K, Lippke S, Nigg CR. Future directions of multiple behavior change research. J Behav Med 2017; 40:194-202.

19. Lima-Costa MF, Andrade FB, Souza Jr. PRB, Neri AL, Duarte YAO, Castro-Costa E, et al. The Brazilian Longitudinal Study of Aging (ELSI-Brazil): objectives and design. Am J Epidemiol 2018; 187:1345-53.

20. Lima-Costa MF, Macinko J, Andrade FB, Souza Jr. PRB, Vasconcellos MTL, Oliveira CM. ELSI-COVID-19 initiative: methodology of the telephone survey on coronavirus in the Brazilian Longitudinal Study of Aging. Cad Saúde Pública 2020; 36 Suppl 3:e00183120.

21. National Institute on Alcohol Abuse and Alcoholism. United States: rethinking drinking. Alcohol and your health; 2010 https://pubs. niaaa.nih.gov/publications/RethinkingDrink ing/Rethinking_Drinking.pdf (acessado em 30/Jun/2020).
22. World Health Organization. Diet, nutrition and the prevention of chronic diseases; 2003. https://apps.who.int/iris/bitstream/ handle/10665/42665/WHO_TRS_916. pdf? sequence $=1$ (acessado em 30/Jun/2020).

23. Matsudo S, Araújo T, Matsudo V, Andrade D, Andrade E, Oliveira L, et al. Questionário Internacional de Atividade Física (IPAQ): estudo de validade e reprodutibilidade no Brasil. Rev Bras Ativ Fís Saúde 2001; 6:5-18.

24. World Health Organization. Global recommendations on physical activity for health; 2010. https://apps.who.int/iris/bitstream/ handle/10665/44399/9789241599979_eng. pdf? sequence $=1$ (acessado em 30/Jun/2020).

25. Hayat K, Rosenthal M, Xu S, Arshed M, Li P, Zhai P, et al. View of Pakistani residents toward coronavirus disease (COVID-19) during a rapid outbreak: a rapid online survey. Int J Environ Res Public Health 2020; 17:3347.

26. Ministério da Saúde. Boletim Epidemiológico Especial 18. Doença pelo coronavírus COVID-19. https://saude.gov.br/images/ pdf/2020/June/18/Boletim-epidemiologicoCOVID-2.pdf (acessado em 29/Jun/2020).

27. Duczmal LH, Almeida ACL, Duczmal DB, Alves CRL, Magalhães FCO, Lima MS, et al. Vertical social distancing policy is ineffective to contain the COVID-19 pandemic. Cad Saúde Pública 2020; 36:e00084420.

28. Cruz CHB. Social distancing in São Paulo State: demonstrating the reduction in cases using time series analysis of deaths due to COVID-19. Rev Bras Epidemiol 2020; 23:e200056.

29. Aquino EML, Silveira IH, Pescarini JM, Aquino R, Souza-Filho JA. Social distancing measures to control the COVID-19 pandemic: potential impacts and challenges in Brazil. Ciênc Saúde Coletiva 2020; 25:2423-46.

30. Liu X, Luo WT, Li Y, Li CN, Hong ZS, Chen $\mathrm{HL}$, et al. Psychological status and behavior changes of the public during the COVID-19 epidemic in China. Infect Dis Poverty 2020; 9:58.

31. Liang M, Gao L, Cheng C, Zhou Q, Patrick J, Heiner K, et al. Efficacy of face mask in preventing respiratory virus transmission: a systematic review and meta-analysis. Travel Med Infect Dis 2020; 36:101751.

32. Sunjaya AP, Jenkins C. Rationale for universal face masks in public against COVID-19. Respirology 2020; 25:678-9.

33. Cheng VC, Wong SC, Chuang VW, So SY, Chen JH, Sridhar S, et al. The role of community-wide wearing of face mask for control of coronavirus disease 2019 (COVID-19) epidemic due to SARS-CoV-2. J Infect 2020; 81:107-14.

34. Lyu W, Wehby GL. Community use of face masks and COVID-19: evidence from a natural experiment of state mandates in the US. Health Aff (Millwood) 2020; 39:1419-25. 
35. Kennedy DM, José G, Wang Y, Pinto O. Modeling the effects of intervention strategies on COVID-19 transmission dynamics. J Clin Virol 2020; 128:104440.

36. Malta DC, Szwarcwald CL, Barros MBA, Gomes CS, Machado IE, Souza-Júnior PRB. The COVID-19 pandemic and the changes in adult Brazilian lifestyles: a cross-sectional study, 2020. Epidemiol Serv Saúde 2020; 29:e2020407.

37. Lesser IA, Nienhuis CP. The impact of COVID-19 on physical activity behavior and well-being of canadians. Int J Environ Res Public Health 2020; 17:3899.

38. Matias T, Dominski FH, Marks DF. Human needs in COVID-19 isolation. J Health Psychol 2020; 25:871-82.

39. Lippi G, Henry BM, Bovo C, Sanchis-Gomar F. Health risks and potential remedies during prolonged lockdowns for coronavirus disease 2019 (COVID-19). Diagnosis (Berl) 2020; 7:85-90.

40. Sullivan AN, Lachman ME. Behavior change with fitness technology in sedentary adults: a review of the evidence for increasing physical activity. Front Public Health 2017; 4:289.

41. Houghton C, Meskell P, Delaney H, Smalle M, Glenton C, Booth A, et al. Barriers and facilitators to healthcare workers' adherence with infection prevention and control (IPC) guidelines for respiratory infectious diseases: a rapid qualitative evidence synthesis. Cochrane Database Syst Rev 2020; 4:CD013582.
42. Szepietowski JC, Matusiak Ł, Szepietowska M, Krajewski PK, Białynicki-Birula R. Face mask-induced itch: a self-questionnaire study of 2,315 responders during the COVID-19 pandemic. Acta Derm Venereol 2020; 100:adv00152.

43. Smereka J, Ruetzler K, Szarpak L, Filipiak KJ, Jaguszewski M. Role of mask/respirator protection against SARS-CoV-2. Anesth Analg 2020; 10.1213/ANE.0000000000004873.

44. Lippke S, Nigg CR, Maddock JE. Healthpromoting and health-risk behaviors: theorydriven analyses of multiple health behavior change in three international samples. Int J Behav Med 2012; 19:1-13.

45. Satre DD, Gordon NP, Weisner C. Alcohol consumption, medical conditions, and health behavior in older adults. Am J Health Behav 2007; 31:238-48.

46. van Rooij B, Bruijn AL, Folmer CR, Kooistra B, Kuiper EK, Brownlee M. et al. Compliance with COVID-19 Mitigation Measures in the United States. PsyArXiv 2020; 22 abr. https:// psyarxiv.com/qymu3/. 


\section{Abstract}

The objective of this study was to evaluate whether healthy behaviours determine the adoption of individual protective measures to fight COVID-19. The data were obtained from the ELSI-COVID-19 initiative, a telephone survey conducted among participants in the Brazilian Longitudinal Study of Aging (ELSI-Brazil), which includes a national sample representative of the population aged 50 years or older. The outcomes evaluated were three protective measures (not having left home in the past week, wearing a mask when leaving home, and sanitizing hands when returning home), and the explanatory variables were health behaviours (smoking, alcohol consumption, consumption of fruits and vegetables, and physical activity). The associations were evaluated by logistic models, considering adjustments for potential confounding factors. A total of 5,827 individuals participated in the analysis; $32.2 \%$ did not leave home in the last week, and among those who left home, 97.5\% used a face mask, and $97.3 \%$ sanitized their hands when they returned home. The practice of physical activity at the recommended levels was associated with a lower chance of not leaving home in the previous week. Ex-smokers were more likely to use a mask, and those who practised physical activity were less likely to adopt this protective measure. Individuals with low-risk alcohol consumption had a higher chance of sanitizing their hands. Actions aimed at increasing the adoption of protective measures to fight the new coronavirus should consider the existence of vulnerable groups, which can be identified by the distribution of other health behaviours in the population.

Coronavirus Infections; Health Surveys; Telephone Interviews; Epidemiologic Factors; Health Behavior

\section{Resumen}

El objetivo de este estudio fue evaluar si los comportamientos saludables determinan la adopción de medidas protectoras individuales para combatir la COVID-19. Los datos se obtuvieron del iniciativa ELSI COVID-19, encuesta telefónica llevada a cabo entre los participantes del Estudio Brasileño Longitudinal del Envejecimiento (ELSI-Brasil), que incluye una muestra nacional representativa de la población con 50 años o más. Los desenlaces evaluados fueron tres medidas protectoras: no haber salido de casa en la última semana, usar mascarilla cuando se salió de casa e higienizar las manos cuando se volvió a casa. Asimismo, las variables explicativas fueron los comportamientos en salud (tabaquismo, consumo de bebidas alcohólicas, consumo de frutas y hortalizas, así como la práctica de actividad física). Las asociaciones fueron evaluadas mediante modelos logísticos, considerando el ajuste por potenciales factores de confusión. Participaron en el análisis 5.827 individuos; un 32,2\% no salió de casa en la última semana, entre quienes salieron de casa un 97,5\% usaron mascarilla facial y un 97,3\% se higienizaron las manos, cuando regresaron al domicilio. La práctica de actividad física en los niveles recomendados estuvo asociada con una menor oportunidad de no salir de casa en la semana anterior. Los exfumadores presentaron una mayor oportunidad de usar mascarilla y aquellos que practicaban actividad física tenían una menor oportunidad de adoptar esta medida protectora. Individuos con un consumo de bajo riesgo de bebidas alcohólicas presentaron una mayor oportunidad de higienizar sus manos. Las acciones que tengan como objetivo el aumento de la adopción de medidas protectoras para combatir al nuevo coronavirus deben considerar la existencia de grupos vulnerables, que pueden ser identificados por la distribución de otros comportamientos en salud dentro de la población.

Infecciones por Coronavírus; Encuestas Epidemiológicas; Entrevista por Teléfono; Conductas Relacionadas com la Salud
Recebido em 06/Jul/2020

Versão final reapresentada em 27/Jul/2020

Aprovado em 07/Ago/2020 